\title{
Number of traps and trap depth position on statistical distribution of random telegraph noise in scaled NAND flash memory
}

Toshihiro Tomita and Kousuke Miyaji

Department of Electrical and Electronic Engineering, Shinshu University, Nagano 380-8553, Japan

E-mail: kmiyaji@shinshu-u.ac.jp

\begin{abstract}
The dependence of random telegraph noise (RTN) amplitude distribution on the number of traps and trap depth position is investigated using three-dimensional Monte Carlo device simulation including random dopant fluctuation (RDF) in a $30 \mathrm{~nm}$ NAND multi level flash memory. The $\Delta V_{\text {th }}$ tail distribution becomes broad at fixed double traps, indicating that the number of traps greatly affects the worst RTN characteristics. It is also found that for both fixed single and fixed double traps, the $\Delta V_{\text {th }}$ distribution in the lowest cell threshold voltage $\left(V_{\text {th }}\right)$ state shows the broadest distribution among all cell $V_{\text {th }}$ states. This is because the drain current flows at the channel surface in the lowest cell $V_{\text {th }}$ state, while at a high cell $V_{\text {th }}$, it flows at the deeper position owing to the fringing coupling between the control gate (CG) and the channel. In this work, the $\Delta V_{\text {th }}$ distribution with the number of traps following the Poisson distribution is also considered to cope with the variations in trap number. As a result, it is found that the number of traps is an important factor for understanding RTN characteristics. In addition, considering trap position in the tunnel oxide thickness direction is also an important factor.
\end{abstract}




\section{Introduction}

Random telegraph noise (RTN) becomes one of the main causes of error in the latest flash memory technology. ${ }^{1-10)}$ RTN is a time-domain drain current $I_{\mathrm{D}}$ or $V_{\text {th }}$ fluctuation induced by the emission/capture of a single electron at a tunnel oxide trap during read operation, as shown in Fig. $1 .^{2-5)}$ RTN amplitude $\left(\Delta V_{\text {th }}\right)$ and its statistical variation becomes worse in advanced flash memories since single-electron behavior largely affects cell characteristics as the capacitance of a floating-gate (FG)-type NAND flash memory cell is reduced. ${ }^{11-15)}$ It is important to clarify the physical origins and the contributions of RTN statistical characteristics to improve statistical model accuracy.

In our previous work, ${ }^{16)} \Delta V_{\text {th }}$ distributions considering a fixed single trap were studied focusing on substrate doping concentration $N_{\mathrm{A}}$ in a $30 \mathrm{~nm}$ NAND flash memory by three-dimensional (3D) Monte Carlo device simulation, as shown in Figs. 2(a) and 2(b). In this simulation random dopant fluctuation (RDF) is also considered by including atomistic doping. ${ }^{17-20)}$ A single electron was randomly located at the channel surface as a trap with widely varying $N_{\mathrm{A}}$ from $1 \times 10^{17}$ to $3 \times 10^{18} \mathrm{~cm}^{-3}$. The statistical model of the RTN in a NAND flash memory is also considered for comparison, which was given by Fukuda et. al. based on the measurement results used for solving Eqs. (1) and (2), ${ }^{1)}$

$$
\begin{aligned}
& f\left(\Delta V_{\mathrm{th}}\right)=\frac{1}{\sigma} \cdot \exp \left(\frac{-\Delta V_{\mathrm{th}}}{\sigma}\right) \\
& \sigma=\alpha \cdot \frac{t_{\mathrm{ox}}}{\sqrt{L_{\mathrm{eff}} W_{\mathrm{eff}}}} \cdot N_{\mathrm{A}}^{0.6}(\mathrm{~V})
\end{aligned}
$$

where $t_{\mathrm{ox}}, N_{\mathrm{A}}, L_{\mathrm{eff}}$, and $W_{\text {eff }}$ are oxide thickness, channel doping concentration and effective channel length and width, respectively. The parameter $\alpha$ is determined by fitting using the measured RTN data. ${ }^{1)}$ This model considers the effect of the RDF in Eq. (2). From the 3D device simulation results in Fig. 2(a), no $\Delta V_{\text {th }}$ tail distribution is observed in the high $-\Delta V_{\text {th }}$ region when $N_{\mathrm{A}}$ is low here and for all ranges $\left(1 \times 10^{17}-1 \times 10^{18} \mathrm{~cm}^{-3}\right)$. While the tail is observed at a high $N_{\mathrm{A}}\left(3 \times 10^{18} \mathrm{~cm}^{-3}\right)$, as shown in Fig. 2(b). Considering that a low $N_{\mathrm{A}}$ is required for NAND flash memories to prevent the leakage current between $p-n$ junctions in the program inhibit cells ${ }^{21-23)}$. The RTN distribution obtained by the 3D Monte Carlo device simulation underestimates the tail distribution in an actual NAND flash memory by simply considering RDF and a single trap. Therefore, to understand the details of the RTN 
characteristics, other factors should be considered at a low $N_{\mathrm{A}}$. In this work, $\Delta V_{\text {th }}$ distributions in a $30 \mathrm{~nm} 2 \mathrm{bit} / \mathrm{cell}$ multi level cell NAND flash memory architecture are investigated focusing on the number of traps and trap depth position. Here, three kinds of policies are considered for the number of traps used in this simulation: fixed single trap, fixed double traps, and Poisson distribution. ${ }^{1)}$

This paper is organized as follows. In Sect. 2, device simulation conditions are explained. In Sect. 3, we show and discuss the results of the number of traps and trap depth position dependence of $\Delta V_{\text {th }}$ distribution for each cell $V_{\text {th }}$ state. Finally, conclusions are given in Sect. 5 .

\section{3D device simulation conditions}

A $30 \mathrm{~nm}, 2$ bit/cell NAND flash memory cell structure is adopted for 3D device simulation used in this work, as shown in Fig. 3(a). ${ }^{16)}$ The coordinates of the channel length, width, and cell height directions are defined as $\mathrm{x}, \mathrm{y}$ and $\mathrm{z}$ respectively, as shown in this figure. Figures 3(b) and 3(c) show the cross-section views of the $\mathrm{x}-\mathrm{z}$ and $\mathrm{y}-\mathrm{z}$ planes, respectively. The $\mathrm{SiO}_{2}$ tunnel oxide thickness $t_{\mathrm{ox}}$, inter-poly dielectric thickness $t_{\mathrm{IPD}}$, and floating gate (FG) height $t_{\mathrm{FG}}$ are 7,10 , and $80 \mathrm{~nm}$, respectively. ${ }^{8)}$ Both the channel width $W$ and length $L_{\mathrm{g}}$ are set at $30 \mathrm{~nm}$, and the source/drain junction depth $x_{\mathrm{j}}$ is set at $10 \mathrm{~nm} . N_{\mathrm{A}}$ is $3 \times 10^{17} \mathrm{~cm}^{-3}$ while the punch-through stopper (PTS) layer is adopted at $30 \mathrm{~nm}$ below the source/drain junction to suppress the excess-short-channel effect. ${ }^{24-28)}$ The PTS doping concentration is $9 \times 10^{17} \mathrm{~cm}^{-3}$.

Figure 4 shows the four cell $V_{\text {th }}$ state distributions of the 2 bit/cell MLC architecture ${ }^{29)}$ in this work. Cell $V_{\text {th }}$ is controlled by changing the initial amount of charges of FG. The range of $V_{\text {th }}$ values is from -2.5 to $3.5 \mathrm{~V}$. The cell $V_{\text {th }}$ state " 11 " is the lowest cell $V_{\text {th }}$ state corresponding to the erase state, and cell $V_{\text {th }}$ state " 10 " is the highest cell $V_{\text {th }}$ state. Over 100 NAND flash cells with an atomistic doping profile ${ }^{17-20)}$ and traps are prepared by Monte Carlo simulation. To emulate the trapped states, a $1 \times 1 \mathrm{~nm}^{2}$ negative surface charge is randomly placed at the channel surface (tunnel oxide/substrate interface) whose charge amount is equals to that of a single electron. $V_{\text {th }}$ is defined as the $V_{\mathrm{CG}}$ value when $I_{\mathrm{D}}$ reaches $W / L_{\mathrm{g}} \times 10^{-7}$ A. $\Delta V_{\text {th }}$ is obtained by subtracting $V_{\text {th }}$ in detrapped state from that in trapped state for each cell $V_{\text {th }}$ state. Here, atomistic doping profiles are the same for both 
the trapped and detrapped states. As can be understood from this simulation procedure, the trap energy levels are not considered for simplicity. ${ }^{3,10)}$ Three cases are assumed for the number of traps used in the Monte Carlo simulation: the fixed single trap, fixed double traps, and Poisson distribution. Cells without traps can be considered for the Poisson distribution where the number of traps is generated along the Poisson distribution. In this case, two trap densities $\left(N_{\text {trap }}\right)$ are considered, $2 \times 10^{10}$ and $2 \times 10^{11} \mathrm{~cm}^{-2}$ for the low and high trap densities, respectively. Figure 5 shows the probability of the number of traps for each $N_{\text {trap. In this work, the RTN model }}{ }^{1)}$ is used for comparison based on Eqs. (1) and (2) with the device parameters used in this work.

\section{Simulation results and discussions}

Figures 6(a) and 6(b) show $\Delta V_{\text {th }}$ distributions with fixed single trap and double traps cases for each cell $V_{\text {th }}$ state. Again, for the single trap, $\Delta V_{\text {th }}$ tails of all cell $V_{\text {th }}$ states are not broad in the high- $\Delta V_{\text {th }}$ region, indicating that for the single trap is underestimation the distribution compared with the measurement-based RTN model. On the other hand, for the double traps, the $\Delta V_{\text {th }}$ tail distributions of all cell $V_{\text {th }}$ states are broad, which shows a similar trend in the high- $\Delta V_{\text {th }}$ region to the RTN model. Another discrepancy between the $3 \mathrm{D}$ device simulation and the RTN model results is that the average $\Delta V_{\text {th }}$ of the $3 \mathrm{D}$ device simulation is much higher than that of the RTN model. This is because all the simulated cells contain RTN traps while cells without RTN traps are the majority actually. Finally, Figs. 6(a) and 6(b) shows that the $\Delta V_{\text {th }}$ distribution of the lowest cell $V_{\text {th }}$ state (state“11") for both single and double traps is the broadest among all of the cell states. In order to explain these results, current distributions near cell $V_{\text {th }}$ at the center of the channel surface $(x=0 \mathrm{~nm})$ for each cell $V_{\text {th }}$ state are investigated, which are shown in Figs. 7(a) and 7(b). As stated in Sect. 2, the amount of channel current near cell $V_{\text {th }}$ is the same for all cell $V_{\text {th }}$ states because $V_{\text {th }}$ is defined as the $V_{\mathrm{CG}}$ value when $I_{\mathrm{D}}$ reaches $W / L_{\mathrm{g}} \times 10^{-7}$ A. From Fig. 7(a), at a low cell $V_{\text {th }}$, current concentrates at the center of the channel surface because the coupling between FG and the channel is dominant. On the other hand, as the cell $V_{\text {th }}$ increases, current flows at both channel edges since the coupling between CG and the channel gradually becomes dominant. ${ }^{30)}$ Moreover, the channel depth dependence on channel current density for each cell $V_{\text {th }}$ state are shown in Figs. 8(a) and 8(b). The 
horizontal $y$-axis is the channel width direction, as shown in Figs. 7(a) and 7(b). The black plot shows the channel current at the channel surface $(z=-1 \mathrm{~nm})$ and the blue one shows that at $7 \mathrm{~nm}$ below the channel surface. From Fig. 8(a), it is found that almost all of the channel currents flow at the channel surface at the low cell $V_{\text {th }}$ state. On the other hand, the channel current at the high cell $V_{\text {th }}$ is less dependent on the channel depth owing the fringing coupling from the CG. Therefore, since the traps are located at the channel surface in this simulation, a high $\Delta V_{\text {th }}$ tends to exist at the low cell $V_{\text {th. Figures }} 9$ (a) and 9(b) show $\Delta V_{\text {th }}$ against trap position along $y$ with double traps for the lowest and highest cell $V_{\text {th }}$ states, respectively. Here, the same atomistic channel doping is used in both Figs. 9(a) and 9(b). The horizontal axis $y_{1}$ shows the trap location along $y$, and vertical axis $y_{2}$ shows the other trap location. From Fig. 9(a), the high- $\Delta V_{\text {th }}$ region at the lowest cell $V_{\text {th }}$ is broader than that at the highest cell $V_{\text {th }}$. Also, from this result, it is found that a high $\Delta V_{\text {th }}$ occurs at the lowest cell $V_{\text {th }}$ in this work.

Figures $10(\mathrm{a})$ and $10(\mathrm{~b})$ show the $\Delta V_{\text {th }}$ distributions for the Poisson distribution with the cell $V_{\text {th }}$ states "11" and "10" for the low and high $N_{\text {trap }}$ values, respectively. In these figures, the average $\Delta V_{\text {th }}$ is closer to the measurement-based model for the Poisson distribution compared with the fixed trap number in Figs. 6(a) and 6(b), since the cells without RTN is included in this trap case. It can also be seen that the simulated distribution seems to be the combination of the distributions of the single trap and double traps from Fig. 10(a) at a low $N_{\text {trap }}$ where the distribution of the single trap is predominant in the low- $\Delta V_{\text {th }}$ region while that of the double traps is predominant in the high- $\Delta V_{\text {th }}$ region. On the other hand, the $\Delta V_{\text {th }}$ distribution at a high $N_{\text {trap }}$ in Fig. 10(b) shows wide variations compared with the RTN model since a significant number of cells contain more than two traps, as shown in Fig. 5 (b). However, this result obviously overestimates the $\Delta V_{\text {th }}$ distribution since not all of the traps will contribute to RTN, as is the case in this simulation. Although there are rooms for the $3 \mathrm{D}$ device simulation to reproduce the measurement-based results, it is clear that the number of traps should be considered to understand RTN characteristics. At least, multiple traps are the significant source of the large tail distribution of the RTN.

In addition, in this work, the dependence on trap position in the tunnel oxide thickness direction is investigated for both the low and high cell $V_{\text {th }}$ states, as shown Fig. 11 . The $1 \times$ $1 \times 1 \mathrm{~nm}^{3}$ negative volume charge is assumed as a charged trap in this simulation. The trap 
is randomly placed $3 \mathrm{~nm}$ from the channel surface. The probability of determined trap depth position is uniformity for simplicity. Simulation conditions are the same as those for the Poisson distribution except for the trap depth position. From this figure, it is found that the $\Delta V_{\text {th }}$ distributions considering the trap position in the tunnel oxide thickness direction for both the low and high cell $V_{\text {th }}$ states are narrower than that of no-considering and more similar to those obtained using the RTN model. The effect of changing the trap depth position is greater for the $\Delta V_{\text {th }}$ distribution in the high- $\Delta V_{\text {th }}$ region. The trap depth position is also one of the important factors for understanding RTN characteristics.

\section{Conclusions}

$\Delta V_{\text {th }}$ distributions are investigated focusing on the number of traps and trap position depth in a $30 \mathrm{~nm}$ NAND multi level flash memory by 3D Monte Carlo device simulation including RDF. As a way for setting trap number, the fixed single trap, fixed double traps and Poisson distribution are assumed in this work. $\Delta V_{\text {th }}$ tails for the fixed single trap are not broadly at high $\Delta V_{\text {th }}$ region compared with the measurement-based results. On the other hand, the $\Delta V_{\text {th }}$ tail distributions with the fixed double traps are broadly like that. Average $\Delta V_{\text {th }}$ values for both of the fixed single trap and double traps are higher than that since are not considered cells without traps. The $\Delta V_{\text {th }}$ distribution in the lowest cell $V_{\text {th }}$ state is the broadest among all cell states because channel current concentrates on the channel surface. Therefore, a high $\Delta V_{\text {th }}$ exists in the lowest cell $V_{\text {th }}$ state since the trap is located at the channel surface. Moreover, the $\Delta V_{\text {th }}$ distribution with the number of traps along the Poisson distribution is investigated. Since no trap cells are considered in this trap, The average $\Delta V_{\text {th }}$ is markedly close to measurement-based results. It is found that the number of traps should be considered to understand RTN characteristics. The trap position in the tunnel oxide thickness direction is investigated. As a result, this factor was found to affect the $\Delta V_{\text {th }}$ distribution in the high- $\Delta V_{\text {th }}$ region for both the low and high cell $V_{\text {th }}$ states and to be similar to measurement-based results.

\section{Acknowledgments}

This work is partly supported by JSPS KAKENHI Grant Number 25820148. The authors 
thank the TCAD academic committee and Professor Hiramoto for help and discussions. 


\section{References}

1) K. Fukuda, Y. Shimizu, K. Amemiya, M. Kamoshida, and C. Hu, IEDM Tech. Dig., 2007, p. 169.

2) K. Takeuchi, T. Nagumo, S. Yokogawa, K. Imai, and Y. Hayashi, Symp. VLSI Tech. Dig., 2009, p. 54.

3) A. Ghetti, C. M. Compagnoni, A. S. Spinelli, and A. Visconti, IEEE Trans. Electron Devices 56, (2009) 1746.

4) S. M. Joe, M. K. Jung, W. Lee, M. S. Lee, B. S. Jo, J. H. Bae, S. K. Park, K. R. Han, J. H. Yi, G. S. Cho, and J. H. Lee, Symp. VLSI Tech. Dig., 2011, 112.

5) C. M. Compagnoni, A. S. Spinelli, S. Beltrami, M. Bonanomi, and A. Visconti, IEEE Electron Device Lett 29, (2008) 941.

6) C. M. Compagnoni, R. Gusmeroli, A. S. Spinelli, and A. Visconti, IEEE Trans. Electron Devices, 55, (2008) 3192.

7) A. Ghetti, C. M. Compagnoni, F. biancardi, A. L. Lacaita, S. Beltrami, L. Chiavarone, A. S. Spinelli, and A. Visconti, IEDM Tech. Dig., 2008, 1.

8) A. Ghetti, S. M. Amoroso, A. Mauri, and C. M. Compagnoni, IMW Tech. Dig., 2011, 1.

9) A. Ghetti, S. M. Amoroso, A. Mauri, and C. M. Conpagnoni, IEEE Trans. Electron Device 59, (2012) 309.

10) T. Nagumo, K. Takeuchi, T. Hase and Y. Hayashi, IEEE IEDM Tech. Dig., 2010, p.28.3.1

11) N. Tega, H. Miki, T. Osabe, A. Kotabe, K. Otsuga, H. Kurata, S. Kamohara, K. Tokami, Y. Ikeda, and R. Yamada, IEDM Tech. Dig., 2006, 1.

12) T. Kim, D. He, R. Porter, D. Rivers, J. Kessenich, and A. Goda, IEEE Electron Device Lett 31, (2010) 153.

13) T. Kim, N. Franklin, C. Srinivasan, P. Kalavade, and A. Goda: IEEE Electron Device Lett 32, (2011) 1183.

14) M. K. Jeong, S. M. Joe, H. J. Kang, K. R. Han, G. Cho, S. K. Park, B. G. Park, and J. H. Lee, Symp. VLSI Tech. Dig., 2013, T154.

15) E. Nowak, J. H. Kim, H. Y. Kown, Y. G. Kim, J. S. Sim, S. H. Lim, D. S. Kim, K. H. Lee, Y. K. Park, J. H. Choi, and C. Chung, Symp. VLSI Tech. Dig., 2012, 21.

16) T. Tomita and K. Miyaji, Jpn. J. Appl. Phys 54, (2015), 04DD02

17) Y. Li, C. H. Hwang, T. Y. Li, and M. H. Han, IEEE Trans. Electron Devices 57, (2010) 
437

18) Y. Li, S. M. Yu, J. R. Hwang, and F. L. Yang, IEEE Trans. Electron Devices 55, (2008) 1449.

19) S. Markov, A. S. M. Zain, B. Cheng, and A. Asenov, SOI Conf., (2012), 1.

20) A. Asenov, IEEE Trans. Electron Devices 45, (1998) 2505.

21) A. Torsi, Y. Zhao, H. Liu, T. Tanzawa, A. Goda, P. Kalavade, and K. Parat, IEEE Trans. Electron Devices 58, (2011) 11.

22) K. D. Suh, B. H. Suh, Y. H. Lim, J. K. Kim, Y. J. Choi, Y. N. Koh, S. S. Lee, S. C. Kwon, B. S. Choi, J. S. yum, J. H. Choi, J. R. Kim, and H. K. Lim, IEEE J. Solid-State Circ 30 (1995) 1149.

23) J. D. Lee, C. K. Lee, M. W. Lee, H. S. Kim, K. C. Park and W. s. Lee, IEEE NVSMW 21, (2006) 31 .

24) Y. Taur, G. J. Hu, R. H. Dennand, L. M. Terman, Y. T. Chung, and K. E. Petrillo, IEEE Trans. Electron Devices 32, (1985) 203.

25) R. R. Troutman, IEEE J. Solid-State Circuits 14, (1979) 383.

26) R. H. Dennard, F. H. Gaensslen, V. L. Rideout, and E. Bassous, IEEE Solid-State and Integrated Circuits 9, (2003) 256.

27) D. Hisamoto, W. C. Lee, J. Kedzierski, and H. Takeuchi, IEEE Trans. Electron Devices 47, (2002) 2320.

28) Z. H. Liu, H. Chenming, J. H. Huang, T. Y. Chan, M. C. Jeng, P. K. Ko, and Y. C. Cheng, IEEE Trans. Electron Devices 40, (2002) 86.

29) S. Tanakamaru, C. Hung, A. Esumi, M. Ito, K. Li, and K. Takeuchi, ISSCC Tech. Dig., 2011, 204.

30) D. Kang, S. Lee, H. M. Park, D. J. Lee, J. Kim, J. Seo, C. Lee, C. Song, C. S. Lee, H. Shin, J. Song, H. Lee, J. H. Choi, and Y. H. Jun: Symp. VLSI Tech. Dig., 2011, 206. 


\section{Figure Captions}

Fig. 1 (Color online) Drain current $I_{\mathrm{D}}$ vs control gate voltage $V_{\mathrm{CG}}$ characteristics for trapped and detrapped state RTNs. $\Delta V_{\text {th }}$ is defined as RTN amplitude.

Fig. 2 (Color online) $\Delta V_{\text {th }}$ distributions considering fixed single trap and RDF at various $N_{\mathrm{A}}$ values. (a) Low $N_{\mathrm{A}}$, (b) High $N_{\mathrm{A}}$.

Fig. 3 (Color online) Simulated $30 \mathrm{~nm}$ NAND flash memory cell structure. (a) Overall cell structure view, (b) cross-sectional view of channel gate length $L_{\mathrm{g}}$ direction at the center of channel width $W$, and (c) cross sectional view of $W$ direction at the center of $L_{\mathrm{g}}$.

Fig. 4 (Color online) $V_{\text {th }}$ distribution and corresponding data symbols in MLC NAND flash memory. ${ }^{13)} V_{\text {th }}$ and initial amount of charge in FG for each program state used in this work are also shown.

Fig. 5 (Color online) Probability of number of traps along Poisson distribution for each $N_{\text {trap. }}$ (a) $N_{\text {trap }}=2 \times 10^{10} \mathrm{~cm}^{-2}$ and (b) $N_{\text {trap }}=2 \times 10^{11} \mathrm{~cm}^{-2}$.

Fig. 6 (Color online) $\Delta V_{\text {th }}$ distribution for (a) fixed single trap case and (b) fixed double traps in each cell $V_{\text {th }}$ state.

Fig. 7 (Color online) Current density profiles at the channel center ( $x=0 \mathrm{~nm}$ ) near (a) the lowest cell $V_{\text {th }}$ state and (b) the highest cell $V_{\text {th }}$ state.

Fig. 8 (Color online) Channel depth dependence on the channel current near cell $V_{\text {th }}$ in the (a) lowest cell $V_{\text {th }}$ state and (b) highest cell $V_{\text {th }}$ state.

Fig. 9 (Color online) Spatial $\Delta V_{\text {th }}$ distribution along trap position along $y$ for double traps in the (a) lowest cell $V_{\text {th }}$ state and (b) highest cell $V_{\text {th }}$ state. On the other hand, trap positions along the channel length direction $x$ are fixed to $11 \mathrm{~nm}$ from the source edge where $\Delta V_{\text {th }}$ is highest. ${ }^{10)}$ 
Fig. 10 (Color online) $\Delta V_{\text {th }}$ distribution for Poisson distribution with (a) $N_{\text {trap }}=2 \times 10^{10} \mathrm{~cm}^{-2}$ and (b) $N_{\text {trap }}=2 \times 10^{11} \mathrm{~cm}^{-2}$

Fig. 11 (Color online) Dependence of the trap position in tunnel oxide thickness direction on $\Delta V_{\text {th }}$ distribution. 


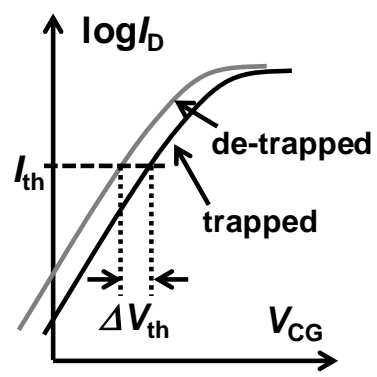

Fig. 1 


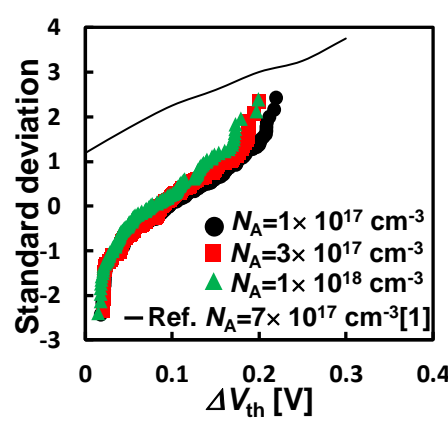

(a)

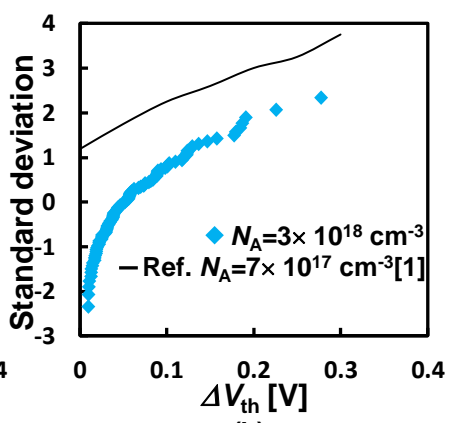

Fig. 2

(b) 


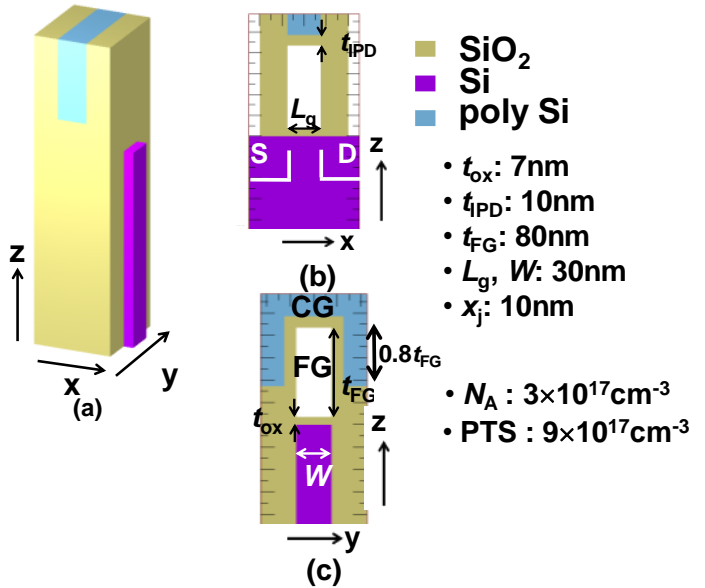

Fig. 3 


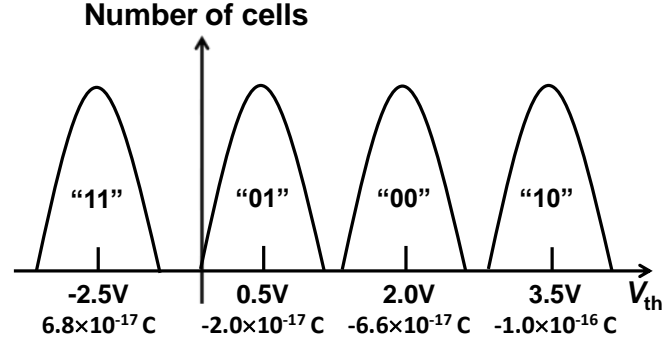

Fig. 4 


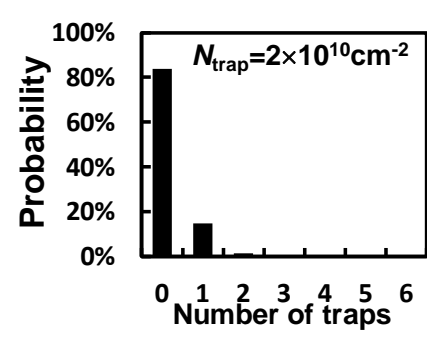

(a)

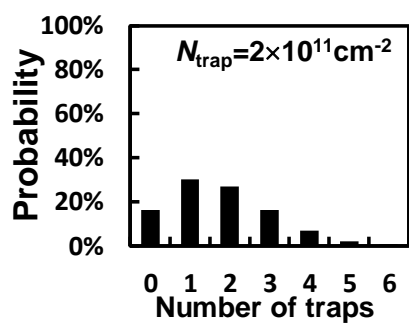

(b)

Fig. 5 

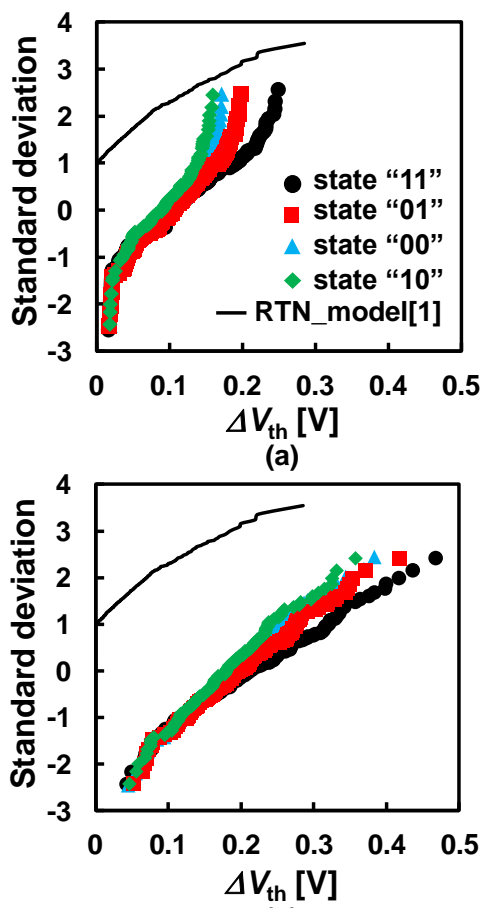

(b)

Fig. 6 


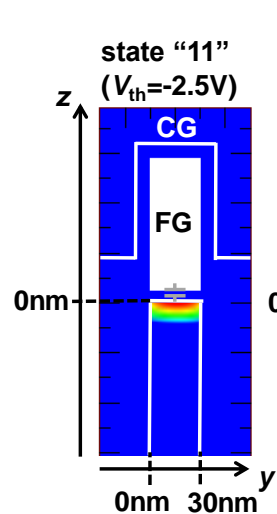

(a)

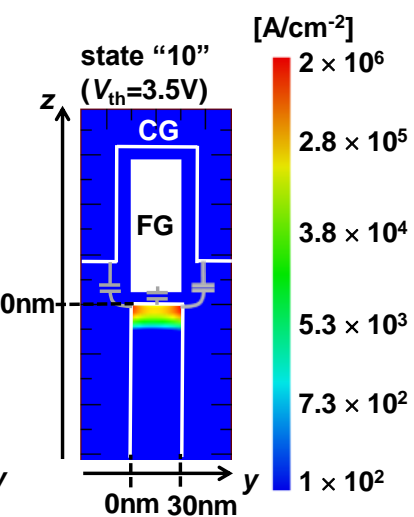

(b)

Fig. 7 


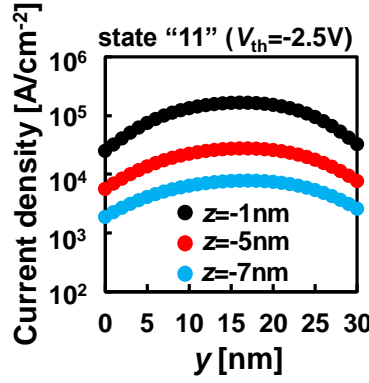

(a)

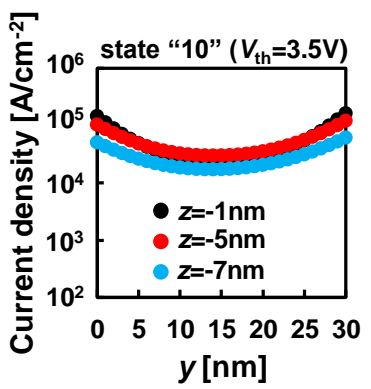

(b)

Fig. 8 


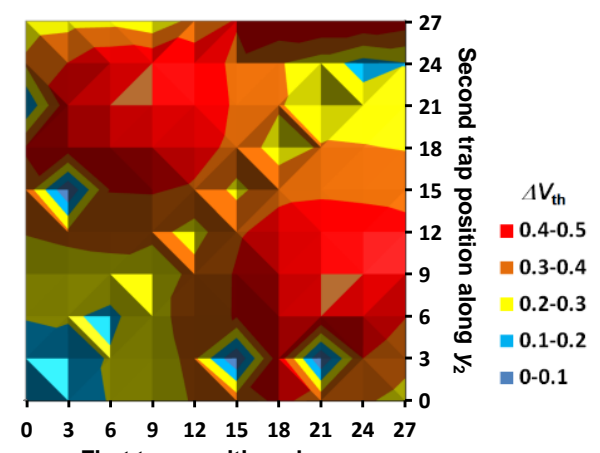

First trap position along $y_{1}$

(a) Low cell $V_{\text {th }}(-2.5 \mathrm{~V})$

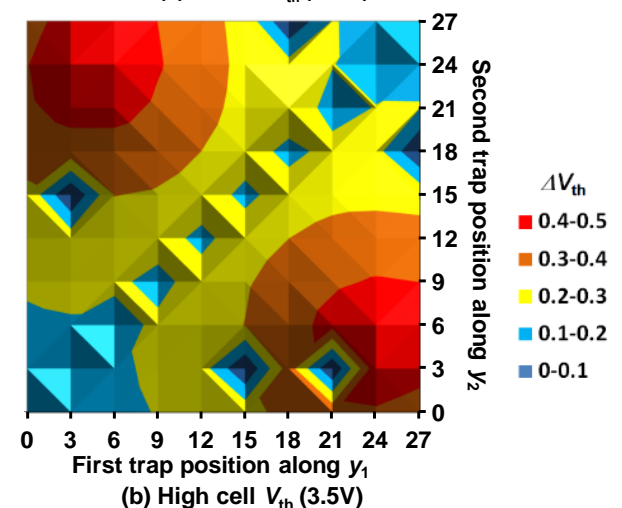

(b) High cell $V_{\text {th }}(3.5 \mathrm{~V})$

Fig. 9 


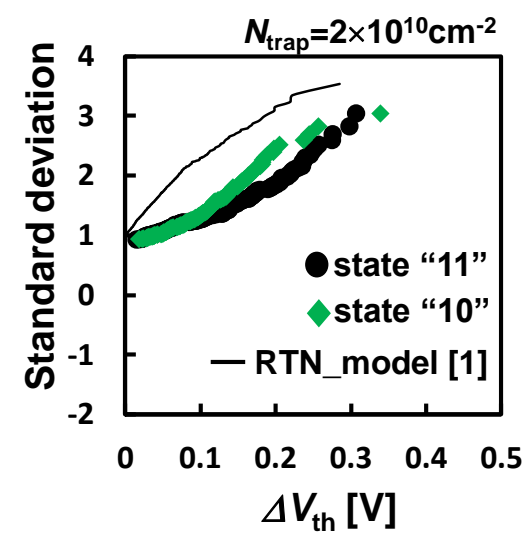

(a) Low trap density

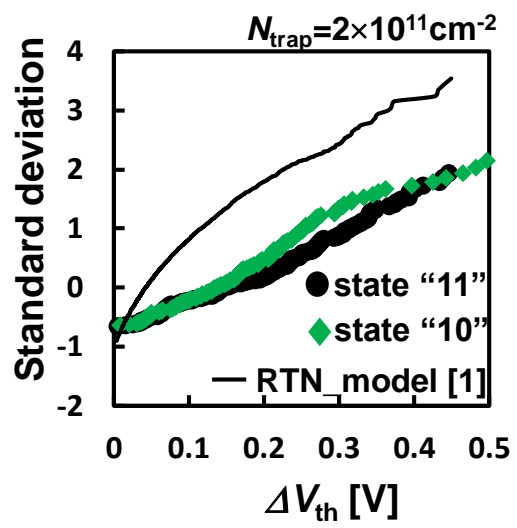

(b) High trap density

Fig. 10 
- trap_depth_no_consider_state"11"

- trap_depth_no_consider_state"10"

- trap_depth_consider_state"11"

- trap_depth_consider_state"10"

- RTN_model [1]

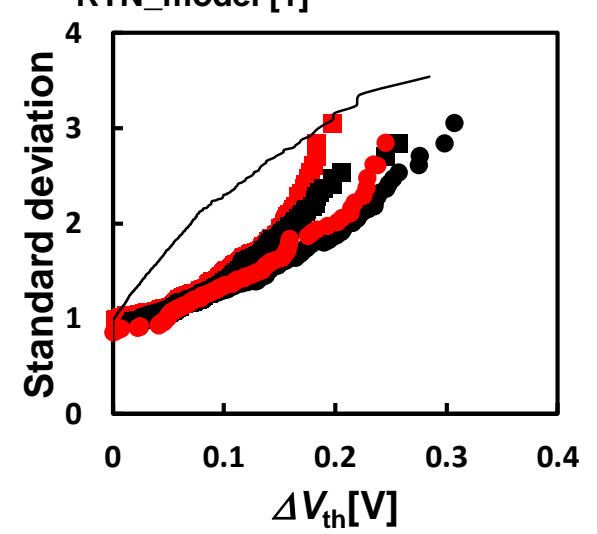

Fig. 11 